\title{
Operant Conditioning of H-Reflex Can Correct a Locomotor Abnormality after Spinal Cord Injury in Rats
}

\author{
Yi Chen, ${ }^{1,2}$ Xiang Yang Chen, ${ }^{1}$ Lyn B. Jakeman, ${ }^{2}$ Lu Chen, ${ }^{1}$ Bradford T. Stokes, ${ }^{2}$ and Jonathan R. Wolpaw ${ }^{1}$ \\ ${ }^{1}$ Laboratory of Nervous System Disorders, Wadsworth Center, New York State Department of Health and State University of New York, Albany, New York \\ 12201, and 2Department of Physiology and Cell Biology, Ohio State University, Columbus, Ohio 43210
}

\begin{abstract}
This study asked whether operant conditioning of the H-reflex can modify locomotion in spinal cord-injured rats. Midthoracic transection of the right lateral column of the spinal cord produced a persistent asymmetry in the muscle activity underlying treadmill locomotion. The rats were then either exposed or not exposed to an H-reflex up-conditioning protocol that greatly increased right soleus motoneuron response to primary afferent input, and locomotion was reevaluated. H-reflex up-conditioning increased the right soleus burst and corrected the locomotor asymmetry. In contrast, the locomotor asymmetry persisted in the control rats. These results suggest that appropriately selected reflex conditioning protocols might improve function in people with partial spinal cord injuries. Such protocols might be especially useful when significant regeneration becomes possible and precise methods for reeducating the regenerated spinal cord neurons and synapses are needed for restoring effective function.
\end{abstract}

Key words: H-reflex conditioning; spinal cord plasticity; motor control; spinal cord injury; locomotion; rehabilitation; learning; memory

\section{Introduction}

The spinal cord, like the rest of the CNS, undergoes activitydependent plasticity in development, during skill acquisition, and in response to trauma and disease (Goode and Van Hoven, 1982; Myklebust et al., 1982, 1986; Casabona et al., 1990; Koceja et al., 1991; O'Sullivan et al., 1991; Nielsen et al., 1993; Straka and Dieringer, 1995; Levinsson et al., 1999; Wolpaw and Tennissen, 2001) (for review, see Wolpaw, 2006). The activity that induces spinal cord plasticity comes from peripheral sensory receptors via dorsal root input pathways and from the brain via descending pathways. Appropriate peripheral input can modify the spinal cord so as to improve motor function after spinal cord injury (for review, see Edgerton et al., 2004; Rossignol et al., 2004). The present study explores the possibility that appropriate descending input can also be used to improve the function of the injured spinal cord.

In normal animals and humans, operant conditioning of the spinal stretch reflex (SSR) (i.e., the tendon jerk) or its electrical analog the $\mathrm{H}$-reflex can modify descending activity and thereby induce spinal cord plasticity that can affect motor function (Wolpaw et al., 1983; Wolpaw, 1987, 2001; Evatt et al., 1989; Chen and Wolpaw, 1995; Segal, 1997; Chen et al., 2005). This

\footnotetext{
Received May 24, 2006; revised Sept. 27, 2006; accepted Sept. 27, 2006.

This work was supported by the Christopher Reeve Paralysis Foundation (X.Y.C.), National Institutes of Health Grants HD36020 (X.Y.C.), NS22189 (J.R.W.), and NS37321 (B.T.S.), the New York State Spinal Cord Injury Trust Fund (X.Y.C.), and the International Spinal Research Trust (J.R.W.). This work constitutes a part of Yi Chen's doctoral thesis presented at the Ohio State University (Columbus, $\mathrm{OH}$ ). We thank Rongliang Liu and Gerwin Schalk for excellent technical assistance and Drs. John A. Buford, Jonathan S. Carp, Dennis J. McFarland, and Elizabeth Winter Wolpaw for valuable advice and comments on this manuscript.

Correspondence should be addressed to Xiang Yang Chen, Laboratory of Nervous System Disorders, Wadsworth Center, New York State Department of Health, P.0. Box 509, Albany, NY 12201-0509. E-mail: chenx@wadsworth.org.

D0I:10.1523/JNEUROSCI.2198-06.2006

Copyright $\odot 2006$ Society for Neuroscience $\quad 0270-6474 / 06 / 2612537-07 \$ 15.00 / 0$
}

suggests that such conditioning might be used to improve defective motor function.

In a first test of this possibility, the present study demonstrates that $\mathrm{H}$-reflex conditioning can correct a physiologically defined locomotor abnormality caused by an anatomically defined spinal cord lesion in rats. Although the full impact on locomotion and the potential clinical significance of this conditioning remain to be determined, the results suggest that reflex conditioning protocols that affect descending activity might provide a precise and practical new method for improving specific aspects of motor function in people with spinal cord injuries or other chronic CNS disorders.

\section{Materials and Methods}

Subjects were 13 female Sprague Dawley rats that were $\sim 90 \mathrm{~d}$ old and weighed 221-257 g (mean \pm SD, $235 \pm 11 \mathrm{~g}$ ) at the beginning of the study. All procedures satisfied the Guide for the Care and Use of Laboratory Animals of the Institute of Laboratory Animal Resources, Commission on Life Sciences, National Research Council (National Academy Press, 1996) and had been reviewed and approved by the Institutional Animal Care and Use Committee of the Wadsworth Center. In each rat, the right lateral column (LC) was transected at the T8-T9 vertebral level. After treadmill locomotion and locomotor $\mathrm{H}$-reflexes were assessed, the rat was (eight HRup rats) or was not (five control rats) exposed to the H-reflex up-conditioning (HRup) protocol, and then treadmill locomotion and locomotor H-reflexes were assessed again. The procedures for LC transection, electrode implantation, treadmill locomotion, $\mathrm{H}$-reflex conditioning, histological evaluation, and data analysis are described have been described in detail previously (Chen and Wolpaw, 1995, 1996, 1997, 2002; Chen et al., 1996, 1999, 2001a, 2002a, 2005) and are summarized here. Figure $1 A$ shows the experimental sequence. Throughout the study, all rats were individually housed in standard rat cages. Laboratory lights were dimmed from 9:00 P.M. to 6:00 A.M. daily.

Electrode implantation. Under anesthesia $(80 \mathrm{mg} / \mathrm{kg}$ ketamine $\mathrm{HCl}$ and $10 \mathrm{mg} / \mathrm{kg}$ xylazine, i.p.), each rat was implanted with chronic stimulating 

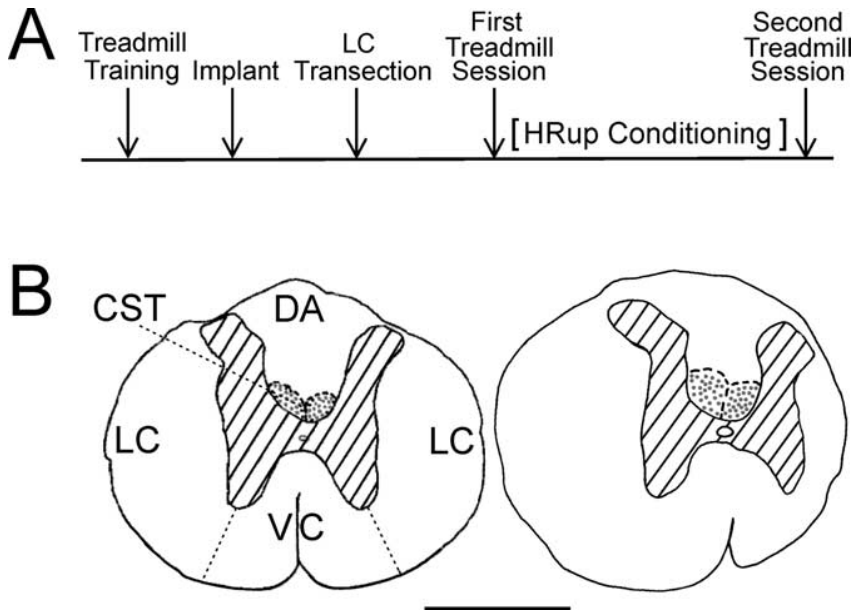

Figure 1. A, Experimental sequence. After learning to walk on the treadmill, each rat was implanted with soleus EMG electrodes and nerve-cuff stimulating electrodes. Several weeks later, it was subjected to an LC transection. After the BBB score (Basso et al., 1995) had returned to 20-21, the first treadmill session assessed locomotion and locomotor H-reflexes. Then, the rats were (HRup rats) or were not (control rats) exposed to the HRup conditioning protocol. Finally, the second treadmill session reassessed locomotion and locomotor H-reflexes. B, Camera lucida drawings of transverse sections of T8-T9 spinal cord. Left, A normal rat with LC, dorsal column CST, dorsal column ascending tract (DA), and ventral column (VC) labeled. Right, An LC-transected rat. In the LC rat, the section shown is at the lesion epicenter. Hatching indicates gray matter, and stippled areas are the main corticospinal tract. Scale bar, $1 \mathrm{~mm}$. Most of the right $\mathrm{LC}$ is gone.

and recording electrodes. To elicit the H-reflex, a nerve cuff was placed on the right posterior tibial nerve just above the triceps surae branches. To record soleus EMG activity, pairs of fine-wire electrodes were placed in the right and left soleus muscles. The wires from the nerve cuff and the muscles were led subcutaneously to a connector plug mounted on the skull. After surgery, the rat was kept warm and given an analgesic (Demerol, $0.2 \mathrm{mg}$, i.m., twice) and was returned to its cage and allowed to eat and drink freely.

$L C$ transection. Two to 4 weeks (mean $\pm \mathrm{SD}, 20 \pm 6 \mathrm{~d}$ ) after electrode implantation, each rat was anesthetized (as described above) for LC transection. A one-vertebra dorsal laminectomy was performed at T8-T9, the cord was visualized with a dissection microscope, and the entire right LC (i.e., the lateral half of the right side of the cord) was transected by electrocautery. This procedure destroyed the rubrospinal, vestibulospinal, and reticulospinal tracts (Tracey, 2004), but it left intact the main corticospinal tract (CST), which is located at the base of the dorsal column in the rat (Tracey, 2004) and is the only major descending path known to be essential for H-reflex conditioning (Chen and Wolpaw, 1997, 2002; Chen et al., 2002a). The site was rinsed and covered with Durafilm to minimize dural adhesions, and the muscle and skin were sutured in layers. The rat was kept warm and received analgesia (see above).

Our standard postinjury care protocol (i.e., bladder management, antibiotics, food, and vitamin supplements) has been described in detail previously (Chen and Wolpaw, 1997, 2002; Chen et al., 1996, 1999, 2002a, 2003). Bladder function returned within $5 \mathrm{~d}$, and rats resumed gaining weight within $22 \mathrm{~d}$. All rats regained their pretransection weights by $29 \mathrm{~d}$ after LC transection, remained healthy and active, and continued to gain weight until the end of study. Body weight increased from $306 \pm$ $24 \mathrm{~g}$ (mean $\pm \mathrm{SD}$ ) just before LC transection to $373 \pm 83 \mathrm{~g}$ at the time of perfusion.

Treadmill locomotion and the locomotor $H$-reflexes. Before implantation surgery, each rat learned to walk quadripedally on a motor-driven treadmill at 9-10 m/min (Burghardt et al., 2004; Chen et al., 2005). After implantation and LC transection, locomotor data were collected in one treadmill session after the Basso, Beattie, and Bresnahan (BBB) score (Basso et al., 1995) had returned to 20-21 and in a second session 49-72 $\mathrm{d}$ after the first. Between these two sessions, the HRup rats underwent up-conditioning of the right soleus $\mathrm{H}$-reflex, whereas the control rats did not. In each rat, treadmill speed was the same for both sessions. During locomotion, EMG was continuously recorded (0.1-1.0 kHz bandpass) from right and left soleus muscles, digitized $(4.0 \mathrm{kHz})$, and stored.

In each treadmill session for each rat, we first recorded right and left soleus EMG during undisturbed locomotion for 300-600 step cycles. The EMG was rectified, low-pass filtered by a $50 \mathrm{~ms}$ running average, and used to calculate the following: step cycle duration (time in seconds between one right burst onset and the next right burst onset); step-cycle length (treadmill speed in centimeters per second times step-cycle duration in seconds); right and left burst amplitudes (EMG area between burst onset and offset); right and left burst widths (burst amplitude divided by its peak value); right and left burst durations (time from burst onset to offset); and step-cycle symmetry. Burst onset was defined as the time at which the rectified EMG reached $10 \%$ of its peak value, and burst offset was defined as the time at which it fell below 10\%. Step-cycle symmetry was defined as the time from right burst onset (RBO) to left burst onset (LBO) divided by the time from RBO to the next RBO (i.e., the time of a full step cycle). Thus, a value of 0.5 indicates that the right/left timing of the step cycle, as assessed by the soleus bursts, was symmetrical. In addition to calculating for each rat in each treadmill session the mean value of each of these measures, we also calculated the coefficient of variation of each measure. This measure assessed the consistency across step cycles of these locomotor measures.

In each treadmill session, after recording undisturbed locomotion, we went on to elicit right soleus $\mathrm{H}$-reflexes during the right stance and swing phases of the step cycle for 300-600 step cycles. As previously described and supported by video recording, the times of soleus burst onset and offset were used to define the stance phase (Chen et al., 2005). By these criteria, stance-phase $\mathrm{H}$-reflexes were elicited just past the middle of the stance phase, and the swing-phase $\mathrm{H}$-reflexes were elicited late in the swing phase. From the rectified EMG, these locomotor H-reflexes were measured as average EMG amplitude in the H-reflex interval minus average background EMG amplitude at the time of stimulation and were expressed in units of average background EMG amplitude (Chen and Wolpaw, 1995, 1996, 1997, 2002; Chen et al., 2005). We measure H-reflex size in this manner because the typically high variability in the form of the rat H-reflex makes a simple peak-to-peak measure inappropriate.

The HRup protocol. During the HRup protocol, the rat continued to live in a standard rat cage and had a $40 \mathrm{~cm}$ flexible cable attached to the skull plug continuously (i.e., $24 \mathrm{~h} / \mathrm{d}$ ). The cable, which allowed the animal to move freely in the cage, carried the wires from the EMG and nerve cuff electrodes to a commutator above the cage that connected to EMG amplifiers and a nerve-cuff stimulation unit. The rat had access to water ad libitum throughout. During the $50 \mathrm{~d}$ exposure to the HRup mode, it obtained most of its food by performing the task described below. Animal well-being was carefully checked several times each day, and body weight was measured weekly.

A computer system continuously monitored $(24 \mathrm{~h} / \mathrm{d})$ right soleus EMG and controlled the nerve-cuff stimulus and a reward (i.e., a $20 \mathrm{mg}$ food pellet). If the absolute value of background (i.e., ongoing) EMG (i.e., equivalent to the full-wave-rectified value) remained within a specified range for a randomly varying $2.3-2.7 \mathrm{~s}$ period, a stimulus pulse (typically $0.5 \mathrm{~ms}$ in duration) was delivered by the nerve cuff. Pulse amplitude was initially set just above M-response (i.e., the direct muscle response to nerve stimulation) threshold and then continuously adjusted by the computer to maintain constant M-response size for the whole period of data collection. Under the control mode, the computer simply measured the absolute value of soleus EMG for $50 \mathrm{~ms}$ following the stimulus. Under the HRup mode, a reward was dispensed $200 \mathrm{~ms}$ after nerve stimulation if EMG in the H-reflex interval (e.g., 5.5-9.5 ms after stimulation) exceeded a criterion value. In the course of its normal activity, the animal usually satisfied the background EMG requirement and thus received nerve-cuff stimulation, 3000-9000 times per day. H-reflex size was calculated as average EMG amplitude in the H-reflex interval minus average background EMG amplitude at the time of stimulation and was expressed in units of average background EMG amplitude. This $\mathrm{H}$-reflex elicited in the conditioning protocol is called "the conditioning 
H-reflex" to distinguish it from the locomotor H-reflexes elicited during the stance and swing phases of locomotion.

Data were collected under the control mode for at least $10 \mathrm{~d}$ to determine the initial size of the rat's conditioning H-reflex. It was then exposed to the HRup mode for $50 \mathrm{~d}$. To determine the final effect of HRup mode exposure on conditioning $\mathrm{H}$-reflex size, the average $\mathrm{H}$-reflex size for the final $10 \mathrm{~d}$ of the exposure was calculated as percentage of initial H-reflex size (i.e., average of final 10 control-mode days). As in the past, successful conditioning was defined as a change of $\geq 20 \%$ in the correct direction (Wolpaw et al., 1993; Chen and Wolpaw, 1995).

Statistical analysis. The primary objective of this study was to determine whether H-reflex up-conditioning, which occurred between the first and second treadmill sessions, affected locomotion and locomotor H-reflexes. To do this, we compared, for the HRup and control rats, the data from the two treadmill sessions by one-way repeated-measures ANOVA, followed (if significant variation was detected) by pairwise multiple comparison (Tukey's test).

Perfusion, postmortem examination, and lesion verification. After data collection was completed, each rat received an overdose of sodium pentobarbital (intraperitoneal) and was perfused through the heart (Chen and Wolpaw, 1997, 2002; Chen et al., 2002a). Nerve cuff, EMG electrodes, and tibial nerve were examined, and soleus muscles of both sides were removed and weighed. Soleus muscle weights (measured as percentage of body weight) were symmetrical and did not differ significantly between HRup and control groups, nor did they differ from soleus muscle weights of normal rats (Chen and Wolpaw, 1995, 1996, 1997, 2002; Chen et al., 2002a, 2005). The spinal cord was removed, and blocks encompassing the transection were embedded in paraffin. Transverse $20-\mu \mathrm{m}$-thick serial sections from the paraffin-embedded blocks were processed and used to determine the location and size of the lesions as described previously (Chen and Wolpaw, 1997, 2002; Chen et al., 2002a).

Figure $1 B$ shows camera lucida drawings of T8-T9 transverse sections from a normal rat (left) and from a rat with right LC transection (right). In the 13 LC-transected rats of this study, $72 \pm 18 \%$ (mean \pm SD) of the right LC was destroyed, whereas other structures were left mostly or totally intact. All of the left LC, $93 \pm 25 \%$ of the right and $96 \pm 13 \%$ of the left dorsal column CST, $76 \pm 28 \%$ of the right and $97 \pm 7 \%$ of the left dorsal column ascending tract, and $91 \pm 22 \%$ of the right and all the left ventral column remained. There was no significant difference between the HRup rats and the control rats in any of these measures $(p>0.2$ for all comparisons by $t$ test).

\section{Results}

\section{Effects of LC transection on the step cycle}

Locomotion, which was markedly impaired immediately after LC transection, improved rapidly in subsequent days. On day 1 , the $\mathrm{BBB}$ score averaged $1 \pm 2($ mean $\pm \mathrm{SD})$ on the right and $12 \pm 2$ on the left. By day 18, the BBB score was 20-21 in both legs. Although all rats scored 21 (i.e., normal) on the left, 12 of 13 scored 20 on the right, reflecting the persistence of some lateral instability on the right (Basso et al., 1995). In the first treadmill session, which was conducted after the BBB score (Basso et al., 1995) had returned to 20-21, all rats walked well on the treadmill at a speed of $9-10 \mathrm{~m} / \mathrm{min}$ [comparable with speeds used for normal rats (Burghardt et al., 2004; Chen et al., 2005)]. Nevertheless, quantitative assessment of treadmill locomotion revealed a persistent locomotor asymmetry.

The LC rats displayed a clear asymmetry in the onset times of the right and left soleus bursts. The time from RBO to LBO was less than the time from $\mathrm{LBO}$ to RBO. The time from RBO to LBO averaged $0.43 \pm 0.02$ (mean \pm SEM) of the step cycle, significantly different ( $p=0.009$ by $t$ test) from the 0.50 characteristic of normal symmetrical locomotion. [For example, in the 11 normal rats of a previous study (Chen et al., 2005), the average value was $0.50 \pm 0.03$ (mean \pm SEM).] Thus, in the LC rats of this study, the time from RBO to LBO averaged only $77 \%$ of the time from LBO to RBO, implying that the right stance phase of locomotion was shortened by the spinal cord injury.

In none of the other locomotor parameters did the LC rats differ significantly from normal rats. At the same time, it should be noted that absolute measures such as soleus burst amplitude differ widely across normal rats and even between the right and left legs of individual rats. This is doubtless attributable in considerable part to the unavoidable differences among rats and between legs in the placements of the soleus EMG electrode pairs. This wide inter-rat variability means that very large groups of LC and normal rats would be needed for intergroup differences to become significant. Of additional note is the fact that for none of the measures did LC rats and normal rats differ significantly in coefficient of variation. Thus, LC transection did not appear to affect the consistency of the step cycle over a treadmill session.

\section{Effects of H-reflex up-conditioning on locomotor H-reflexes and soleus locomotor bursts}

H-reflex up-conditioning was successful [i.e., increase $\geq 20 \%$ from control H-reflex size (Wolpaw et al., 1993; Chen and Wolpaw, 1995)] in seven of the eight rats exposed to the HRup protocol (i.e., success rate of $87.5 \%$ ). In these rats, the size of the conditioning H-reflex (i.e., the H-reflex elicited by the conditioning protocol) rose to $212 \pm 49 \%$ (mean \pm SEM) of control, whereas background EMG and M-response size did not change. The success rate and average final increase were comparable with previous values for normal rats and LC rats (Chen and Wolpaw, 1995,1997, 2002; Chen et al., 1996, 1999, 2001b, 2002a,b; Carp et al., 2001) and thus further confirmed that LC transection does not impair H-reflex up-conditioning. The seven successful rats constituted the experimental group of HRup rats for evaluation of the effects of up-conditioning on treadmill locomotion. The five rats not exposed to up-conditioning constituted the control group.

To assess the effects of H-reflex up-conditioning on locomotor H-reflexes and soleus locomotor bursts, we compared (see Materials and Methods) the data from the first and second treadmill sessions. Figure $2 \mathrm{~A}$ summarizes the results for the sizes of the right soleus locomotor $\mathrm{H}$-reflexes and for the amplitudes, widths, and durations of the right and left soleus locomotor bursts. In the control group, the results for the second session are not significantly different from those of the first session. In contrast, in the HRup group, the locomotor H-reflexes and the right soleus burst amplitudes and widths are significantly larger in the second session than in the first $(p<0.001, p=0.02$, and $p=0.03$, respectively).

As in normal rats (Chen et al., 2005), the increase in the stance H-reflex in the HRup group of LC rats was comparable with the increase in the conditioning $\mathrm{H}$-reflex [i.e., the $\mathrm{H}$-reflex elicited by the conditioning protocol; increases to $280 \pm 38 \%$ (mean \pm SEM) and $212 \pm 49 \%$ for the stance $\mathrm{H}$-reflex and the conditioning H-reflex, respectively]. At the same time, the right soleus burst amplitude increased to $158 \pm 22 \%$ (mean \pm SEM). On average, an increase in the stance $\mathrm{H}$-reflex was accompanied by an increase 0.32 times as large in soleus burst amplitude $(r=0.60$; $p=0.04$ ). This relationship is consistent with previous results from normal rats (Chen et al., 2005) and with other data on the contribution of primary afferent input to the locomotor burst (Yang et al., 1991; Bennett et al., 1996; Stein et al., 2000). The increases in burst amplitude and width are likely to reflect an increase in the number of motoneurons responding to this input. The width increase also suggests that after up-conditioning the individual motoneurons tend to fire more action potentials. That 

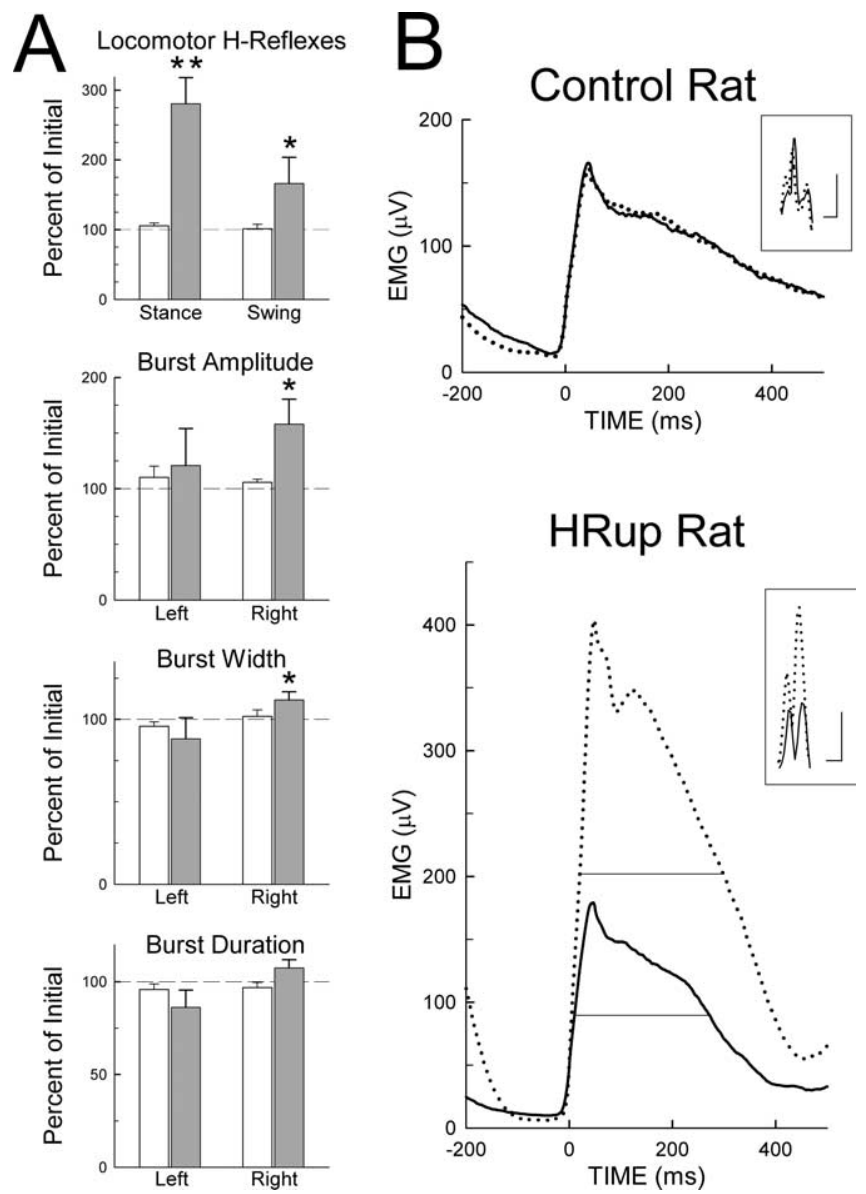

Figure 2. Effects of H-reflex up-conditioning on locomotor H-reflexes and soleus locomotor bursts. $A$, Average \pm SEM locomotor H-reflexes and left and right soleus burst amplitudes, widths, and durations for control rats (open bars) and HRup rats (solid bars) for the second treadmill session as percentage of their values for the first (i.e., initial) session. Asterisks indicate significant changes from the first to the second session $\left({ }^{*} p<0.05\right.$; $\left.{ }^{* *} p<0.01\right)$. Locomotor $\mathrm{H}$-reflexes and right soleus burst amplitudes and widths are increased in the HRup rats. The control rats show no significant change. $\boldsymbol{B}$, The main traces are average right soleus bursts and the inset small traces are average stance $\mathrm{H}$-reflexes from a control rat and an HRup rat for the first (solid) and second (dotted) treadmill sessions. [For the H-reflexes shown, average background EMG and M-response size were the same for the first and second sessions (for methods, see Chen et al., 2005).] Calibration: horizontal bars, 2 ms for both the control and HRup rats; vertical bars, 50 and $200 \mu \mathrm{V}$, respectively. The fine horizontal lines in the bottom graph indicate burst half-width (i.e., the width at half the peak value). (Half-width is shown here only for illustrative purposes. Materials and Methods describes the more comprehensive measure used to assess the effects of conditioning on burst width.) In the second session, the stance H-reflex and the right soleus burst amplitude and half-width are increased in the HRup rat. The control rat shows no change in the $\mathrm{H}$-reflex or in the burst.

these H-reflex and locomotor burst increases in the HRup rats were specific effects of the HRup protocol is indicated by a previous study (Chen et al., 2001a) showing the long-term stability of H-reflex size in unconditioned LC rats and a recent study (Chen et al., 2005) showing that H-reflex up- and downconditioning each has its expected effect on soleus burst amplitude (i.e., increase and decrease, respectively).

Although the HRup rats showed no significant change in total duration of the right soleus burst, the significant increase in burst width indicates that the burst became wider as well as larger. That is, the increase in burst amplitude was greater than that accounted for by the increase in its peak value: the soleus EMG both increased and became more broadly distributed.


Figure 3. Effects of H-reflex up-conditioning on the step-cycle. $A$, Average \pm SEM stepcycle duration and RBO-LBO duration (time from right soleus burst onset to left soleus burst onset) for control rats (open bars) and HRup rats (solid bars) for the second treadmill session as percentage of their values for the first (i.e., initial) session. ${ }^{* *} p<0.01$, significant change from the first to the second session. Step-cycle duration is unchanged in both control and HRup rats. RBO-LBO duration (which was lower than normal in the first treadmill session; see Results) is increased in HRup rats only. $\boldsymbol{B}$, Right and left soleus bursts (rectified EMG) from an HRup rat for the first (i.e., before up-conditioning) treadmill session and the second (i.e., after upconditioning) session. Calibration: horizontal bar, $0.5 \mathrm{~s}$; vertical bar, 100 and $150 \mu \mathrm{V}$ for the right and left bursts, respectively. $\operatorname{Each} \mathrm{RBO}(-)$ or $\mathrm{LBO}(\bigcirc)$ is marked. The short vertical dashed lines mark the midpoints between RBOs (i.e., the midpoints of the step-cycles), which is the time when LBOs should occur (as in normal rats). Before H-reflex up-conditioning, LBO occurs too early; after up-conditioning, it occurs on time.

In both HRup and control groups, the coefficients of variation for soleus burst amplitude, width, and duration did not differ significantly between the first and second treadmill sessions.

Figure $2 B$ illustrates the effects of up-conditioning on the stance H-reflex and on the right burst amplitude and width. In the HRup rat, the H-reflex and the burst are much larger and the burst is broader in the second session. In the control rat, the H-reflex and the burst do not change from the first to the second session.

\section{Effects of H-reflex up-conditioning on the step cycle}

Figure $3 A$ summarizes the effects of up-conditioning on stepcycle duration and on the right/left asymmetry in the step-cycle present in the first session. Step-cycle duration did not change in the HRup group or the control group. Because for each rat treadmill speed was the same in the two sessions, this result also indicates that the length of the step-cycle (i.e., right stride plus left stride) did not change. For neither step-cycle duration nor right/ left asymmetry did the coefficient of variation change from the first to the second treadmill session. 
However, as Figure $3 \mathrm{~A}$ indicates, the right/left asymmetry mean values were different for the control and HRup groups. In the control group, the right/left asymmetry persisted in the second session: the time from RBO to LBO remained shorter than the time from LBO to RBO. In contrast, in the HRup group, the asymmetry disappeared ( $p=0.008$ ). Figure $3 B$ illustrates the elimination of the asymmetry in an HRup rat. It shows right and left soleus bursts before and after up-conditioning, with each RBO (O) and LBO $(\bigcirc)$ marked. The short vertical dashed lines mark the midpoints between successive RBOs, which is the time when LBO should occur (as it does in normal rats). Before upconditioning, LBO occurs too early; after up-conditioning, it occurs on time.

\section{Discussion}

Right LC transection created a clear locomotor abnormality that was evident in both HRup and control LC rats in the first treadmill session. The time from RBO to LBO was substantially shorter than the time from LBO to RBO. In light of evidence for the close relationship between the onset of the soleus burst and the onset of the stance phase of locomotion (Chen et al., 2005), this timing asymmetry implies that right LC rats did not sustain right stance normally (i.e., they limped).

In the second treadmill session, this locomotor asymmetry was present only in the control rats. In the HRup rats, symmetry was restored: the time from $\mathrm{RBO}$ to $\mathrm{LBO}$ equaled that from LBO to RBO. The correspondence between soleus and gastrocnemius activity and the similar although smaller effect that soleus $\mathrm{H}$-reflex conditioning has on the gastrocnemius H-reflex (Chen et al., 2005) imply that this restored symmetry occurs also in stance onset. The probable origin is the marked increase in locomotor burst amplitude that results from H-reflex upconditioning (i.e., Fig. 2). As noted above, this increase is consistent with the expected increased contribution of primary afferent input to the burst (Yang et al., 1991; Bennett et al., 1996; Stein et al., 2000; Chen et al., 2005). The increase in right burst width could also contribute to the restoration of symmetry.

HRup conditioning increases locomotor H-reflexes and the ipsilateral soleus locomotor burst in normal rats (Chen et al., 2005) just as it does in the LC rats of the present study. In normal rats, however, neither HRup nor HRdown conditioning affects right/left symmetry: the time from $\mathrm{RBO}$ to $\mathrm{LBO}$ remains equal to the time from $\mathrm{LBO}$ to RBO, and it is not longer in normal HRup rats or shorter in normal HRdown rats. In normal rats, it is likely that adjustments by other muscles, and possibly additional adaptive activity-dependent plasticity, compensate for the change in the soleus locomotor burst so as to preserve normal locomotor symmetry (Chen et al., 2005). In contrast, when a locomotor asymmetry is already present before $\mathrm{H}$-reflex conditioning, as it is in the LC rats of this study, the same automatic adjustments and the same impetus for adaptation to eliminate the impact of conditioning on locomotion are likely to be impaired or entirely absent. Indeed, in LC rats, H-reflex up-conditioning is itself adaptive because it restores locomotor symmetry. Thus, it is explicable that, in LC rats, unlike in normal rats, H-reflex upconditioning actually changes locomotion.

These results suggest that $\mathrm{H}$-reflex conditioning could help to restore function to people with partial spinal cord injuries who retain a functioning CST, which appears to be the only major descending trace essential for H-reflex conditioning (Chen and Wolpaw, 1997, 2002; Chen et al., 2002a). This hypothesis is supported by evidence that $\mathrm{H}$-reflex conditioning is possible in rats with substantial spinal cord contusion injuries and that biceps
SSR conditioning is possible in people with partial spinal cord injuries (Segal and Wolf, 1994; Chen et al., 1996, 1999) [and is not possible in people with strokes involving sensorimotor cortex, the principal origin of the CST (Segal, 1997)]. Furthermore, human data suggest that the thousands of trials per day obtained from rats are not essential and that conditioning can be achieved with three to five sessions per week of several hundred trials each (Segal and Wolf, 1994; Thompson et al., 2006).

At the same time, however, it must be recognized that the present results merely validate the principle that spinal reflex conditioning can affect a complex motor function after spinal cord injury. Much remains to be learned before the potential clinical significance of this principle can be properly assessed. The data presented here rely on the right and left soleus locomotor bursts to assess specific aspects of locomotion. Although these measures appear to parallel the right and left stance phases of locomotion (Chen et al., 2005), future studies need to measure the stance and swing phases more directly and to assess other aspects of limb movement during locomotion, as well as the activity of the other muscle groups that contribute to locomotion. It is highly probable that conditioning affects these other measures as well. Such effects could come directly from the plasticity underlying H-reflex conditioning and/or could be secondary effects triggered by the changes in soleus activity (Wolpaw and Tennissen, 2001; Chen et al., 2005). Most important, the practical functional impact of reflex conditioning requires assessment. For example, it is not as yet clear whether the soleus EMG asymmetry created by the LC lesion is associated with an actual limp in walking or whether there is a limp and whether it, like the EMG asymmetry, is corrected by up-conditioning. These issues become more critical and complex in considering the application of spinal reflex conditioning to humans with spinal cord injuries and in determining the degree to which the effects noted in a simple highly controlled laboratory environment persist outside the laboratory. The extent to which the effects of conditioning on treadmill locomotion in the quadripedal rat predict its effects on over-ground locomotion in the bipedal human remains to be determined.

Furthermore, the long-term persistence of the effects of $\mathrm{H}$-reflex conditioning is not yet well defined. In humans with partial spinal cord injuries, the effects of down-conditioning are still evident 4 months later (Segal and Wolf, 1994). In normal monkeys, the effects of SSR up-conditioning decline with a halflife of $\sim 17 \mathrm{~d}$, whereas the effects of down-conditioning appear to last considerably longer (Wolpaw et al., 1986). It is possible that the functional effects of $\mathrm{H}$-reflex conditioning might require periodic reinforcement by reexposure to the conditioning protocol. Conversely, in individuals with partial spinal cord injuries in which conditioning has improved locomotion, the improved locomotion itself might be sufficient to ensure the persistence of the adaptive reflex change.

The two sources of activity-dependent spinal cord plasticity are sensory inputs from the periphery and descending inputs from the brain. Many studies in animals and humans indicate that the patterned sensory inputs induced by locomotor training regimens can induce spinal cord plasticity and improve function after spinal cord injury (for review, see Edgerton et al., 2004; Rossignol et al., 2004; Dobkin et al., 2006). The present study shows that an operant conditioning protocol can produce descending input that also induces spinal cord plasticity and modifies function.

These two new therapeutic approaches might conceivably complement each other. Although a locomotor training regimen 
creates a complex stereotyped pattern of sensory inputs that is aimed at restoring a complex behavior (i.e., locomotion), operant conditioning protocols can focus on changing specific spinal cord pathways, that is, the individual elements that underlie and support the complex behavior. Furthermore, as the present results demonstrate, the effects of an operant conditioning protocol are not necessarily limited to the protocol: they can also affect the CNS activity underlying another motor behavior, specifically locomotion.

Conditioning protocols might be designed to address the particular deficits of individual patients. H-reflex conditioning offers several possibilities (e.g., up- or down-conditioning of right or left soleus/gastrocnemius or tibialis anterior muscles). In people with inadequate stance, H-reflex up-conditioning of soleus/ gastrocnemius might be used to strengthen stance (as in the present study), whereas in people with hyperactive reflexes that disturb the step cycle, H-reflex down-conditioning might help to reduce the disturbance. Reciprocal inhibition can also be conditioned (Chen et al., 2006). This may provide additional possibilities for reducing functional deficits. Reflex conditioning protocols could be largely automated so as to require minimal therapist involvement. Moreover, they might possibly be used to address other functional abnormalities associated with spasticity (St. George, 1993; Boorman et al., 1996; Hiersemenzel et al., 2000) or even disorders of autonomic function (Colachis, 1992; Weaver et al., 2002).

The ability to target specific reflex pathways and deficits could be particularly important once significant spinal cord regeneration becomes possible (Schwab and Bartholdi, 1996; McTigue et al., 2000; Selzer, 2003; Liverman et al., 2005). A partially or even wholly regenerated spinal cord will probably not support effective function immediately. Methods for inducing adaptive plasticity that restores effective function will probably be essential. In this context, H-reflex conditioning and comparable conditioning of other simple reflexes could help to shape spinal cord function so as to maximize the recovery of motor control.

In summary, this study shows that $\mathrm{H}$-reflex conditioning can correct a specific locomotor abnormality in spinal cord-injured rats. The full effects, functional impact, and potential clinical significance of such conditioning remain to be defined. Nevertheless, these initial results suggest that operant conditioning protocols might be used to modify aspects of the locomotor and other functional abnormalities associated with spinal cord injuries or other chronic disorders of motor control and might thereby help to restore more effective function.

\section{References}

Basso DM, Beattie MS, Bresnahan JC (1995) A sensitive and reliable locomotor rating scale for open field testing in rats. J Neurotrauma 12:1-21.

Bennett DJ, De Serres SJ, Stein RB (1996) Gain of the triceps surae stretch reflex in decerebrate and spinal cats during postural and locomotor activities. J Physiol (Lond) 496:837-850.

Boorman GI, Lee RG, Becker WJ, Windhorst UR (1996) Impaired "natural reciprocal inhibition" in patients with spasticity due to incomplete spinal cord injury. Electroencephalograph Clin Neurophysiol 101:84-92.

Burghardt PR, Fulk LJ, Hand GA, Wilson MA (2004) The effects of chronic treadmill and wheel running on behavior in rats. Brain Res 1019:84-96.

Carp JS, Chen XY, Sheikh H, Wolpaw JR (2001) Operant conditioning of rat H-reflex affects motoneuron axonal conduction velocity. Exp Brain Res 136:269-273.

Casabona A, Polizzi MC, Perciavalle V (1990) Differences in H-reflex between athletes trained for explosive contraction and non-trained subjects. Eur J Appl Physiol 61:26-32.

Chen XY, Wolpaw JR (1995) Operant conditioning of H-reflex in freely moving rats. J Neurophysiol 73:411-415.
Chen XY, Wolpaw JR (1996) Reversal of H-reflex operant conditioning in the rat. Exp Brain Res 112:58-62.

Chen XY, Wolpaw JR (1997) Dorsal column but not lateral column transection prevents down-conditioning of $\mathrm{H}$ reflex in rats. J Neurophysiol 78:1730-1734.

Chen XY, Wolpaw JR (2002) Probable corticospinal tract control of spinal cord plasticity in the rat. J Neurophysiol 87:645-652.

Chen XY, Wolpaw JR, Jakeman LB, Stokes BT (1996) Operant conditioning of H-reflex in spinal cord-injured rats. J Neurotrauma 13:755-766.

Chen XY, Wolpaw JR, Jakeman LB, Stokes BT (1999) Operant conditioning of H-reflex increase in spinal cord-injured rats. J Neurotrauma 16:175-186.

Chen XY, Feng-chen KC, Chen L, Stark DM, Wolpaw JR (2001a) Shortterm and medium-term effects of spinal cord tract transections on soleus H-reflex in freely moving rats. J Neurotrauma 18:313-327.

Chen XY, Chen L, Wolpaw JR (2001b) Time course of H-reflex conditioning in the rat. Neurosci Lett 302:85-88.

Chen XY, Carp JS, Chen L, Wolpaw JR (2002a) Corticospinal tract transection prevents operantly conditioned H-reflex increase in rats. Exp Brain Res 144:88-94.

Chen XY, Chen L, Wolpaw JR, Jakeman LB (2002b) Corticospinal tract transection reduces H-reflex circadian rhythm in rats. Brain Res 942:101-108.

Chen XY, Chen L, Wolpaw JR (2003) Conditioned H-reflex increase persists after transection of the main corticospinal tract in rats. J Neurophysiol 90:3572-3578.

Chen XY, Chen L, Chen Y, Wolpaw JR (2006) Operant conditioning of reciprocal inhibition in rat soleus muscle. J Neurophysiol 96:2144-2150.

Chen Y, Chen XY, Jakeman LB, Schalk G, Stokes BT, Wolpaw JR (2005) The interaction of a new motor skill and an old one: H-reflex conditioning and locomotion in rats. J Neurosci 25:6898-6906.

Colachis III SC (1992) Autonomic hyperreflexia with spinal cord injury. J Am Paraplegia Soc 15:171-186.

Dobkin B, Apple D, Barbeau H, Basso M, Behrman A, Deforge D, Ditunno J, Dudley G, Elashoff R, Fugate L, Harkema S, Saulino M, Scott M, Spinal Cord Injury Locomotor Trial Group (2006) Weight-supported treadmill vs over-ground training for walking after acute incomplete SCI. Neurology 66:484-493.

Edgerton VR, Tillakaratne NJK, Bigbee AJ, de len RD, Roy RR (2004) Plasticity of the spinal neural circuitry after injury. Annu Rev Neurosci 27:145-167.

Evatt ML, Wolf SL, Segal RL (1989) Modification of human spinal stretch reflexes: preliminary studies. Neurosci Lett 105:350-355.

Goode DJ, Van Hoven J (1982) Loss of patellar and Achilles tendon reflex in classical ballet dancers. Arch Neurol 39:323.

Hiersemenzel L-P, Curt A, Dietz V (2000) From spinal shock to spasticity: neuronal adaptations to a spinal cord injury. Neurology 54:1574-1582.

Koceja DM, Burke JR, Kamen G (1991) Organization of segmental reflexes in trained dancers. Int J Sports Med 12:285-289.

Levinsson A, Luo XL, Holmberg H, Schouenborg J (1999) Developmental tuning in a spinal nociceptive system: effects of neonatal spinalization. J Neurosci 19:10397-10403.

Liverman CT, Altevogt BM, Joy JE, Johnson RT (eds) (2005) Spinal cord injury, progress, promise, and priorities. Washington, DC: National Academies.

McTigue DM, Popovich PG, Jakeman LB, Stokes BT (2000) Strategies for spinal cord injury repair. Prog Brain Res 128:3-8.

Myklebust BM, Gottlieb GL, Penn RL, Agarwal GC (1982) Reciprocal excitation of antagonistic muscles as a differentiating feature in spasticity. Ann Neurol 12:367-374.

Myklebust BM, Gottlieb GL, Agarwal GC (1986) Stretch reflexes of the normal human infant. Dev Med Child Neurol 28:440-449.

Nielsen J, Crone C, Hultborn H (1993) H-reflexes are smaller in dancers from the Royal Danish Ballet than in well-trained athletes. Eur J Appl Physiol 66:116-121.

O'Sullivan MC, Eyre JA, Miller S (1991) Radiation of phasic stretch reflex in biceps brachii to muscles of the arm in man and its restriction during development. J Physiol (Lond) 439:529-543.

Rossignol S, Brustein E, Bouyer L, Barthelemy D, Langlet C, Leblond H (2004) Adaptive changes of locomotion after central and perferal lesions. Can J Physiol Pharmacol 82:617-627. 
Schwab ME, Bartholdi D (1996) Degeneration and regeneration of axons in the lesioned spinal cord. Physiol Rev 76:319-370.

Segal RL (1997) Plasticity in the central nervous system: operant conditioning of the spinal stretch reflex. Top Stroke Rehabil 3:76-87.

Segal RL, Wolf SL (1994) Operant conditioning of spinal stretch reflex in patients with spinal cord injuries. Exp Neurol 130:202-213.

Selzer ME (2003) Promotion of axonal regeneration in the injured CNS. Lancet Neurol 2:157-166.

St. George CL (1993) Spasticity: mechanisms and nursing care. Nurs Clin North Am 28:819-827.

Stein RB, Misiaszek JE, Pearson KG (2000) Functional role of muscle reflexes for force generation in the decerebrate walking cat. J Physiol (Lond) 525:781-791.

Straka H, Dieringer N (1995) Spinal plasticity after hemilabyrinthectomy and its relation to postural recovery in the frog. J Neurophysiol 73:1617-1631.

Thompson AK, Chen XY, Carp JS, Wolpaw JR (2006) Operant conditioning of the soleus H-reflex in humans. Soc Neurosci Abstr 32:146.9.

Tracey DJ (2004) Ascending and descending pathways in the spinal cord. In: The rat nervous system (Paxinos G, ed), pp 149-164. San Diego: Academic.

Weaver LC, Marsh DR, Gris D, Meakin SO, Dekaban GA (2002) Central mechanisms for autonomic dysreflexia after spinal cord injury. Prog Brain Res 137:83-95.

Wolpaw JR (1987) Operant conditioning of primate spinal reflexes: the H-reflex. J Neurophysiol 57:443-459.

Wolpaw JR (2001) Spinal cord plasticity in the acquisition of a simple motor skill. In: Spinal cord plasticity alterations in reflex function (Patterson MM, Grau JW, eds), pp 101-125. Boston: Kluwer Academic.

Wolpaw JR (2006) Plasticity in the intact spinal cord. In: Textbook of neural repair and rehabilitation (Seizer ME, Cohen L, Gage FH, Clarke S, Duncan P, eds). Cambridge: Cambridge UP.

Wolpaw JR, Tennissen AM (2001) Activity-dependent spinal cord plasticity in health and disease. Annu Rev Neurosci 24:807-843.

Wolpaw JR, Braitman DJ, Seegal RF (1983) Adaptive plasticity in the primate spinal stretch reflex: initial development. J Neurophysiol 50:1296-1311.

Wolpaw JR, O’Keefe JA, Noonan PA, Sanders MG (1986) Adaptive plasticity in primate spinal stretch reflex: persistence. J Neurophysiol 55:272-279.

Wolpaw JR, Herchenroder PA, Carp JS (1993) Operant conditioning of the primate H-reflex: factors affecting the magnitude of change. Exp Brain Res 97:31-39.

Yang JF, Stein RB, James KB (1991) Contribution of peripheral afferents to the activation of the soleus H-reflex in walking in humans. Exp Brain Res $87: 679-687$. 